\title{
Internal Friction Due to Long-Range Diffusion of \\ Hydrogen in the Nb-46 wt.\% Ti Alloy
}

\author{
Carlos Roberto Grandini ${ }^{\mathrm{a} *}$, Odila Florêncio ${ }^{\mathrm{b}}$, José Alberto Rodrigues Jordão $^{\mathrm{c}}$ \\ ${ }^{a}$ Departamento de Física and Centro Virtual de Pesquisa em Materiais (CVMat), \\ UNESP 17033-360 Bauru - SP, Brazil \\ ${ }^{\mathrm{b}}$ Departamento de Física, UFSCar, \\ 13565-905 São Carlos - SP, Brazil \\ ${ }^{\mathrm{c}}$ Centro de Caracterização e Desenvolvimento de Materiais, UFSCar, UNESP \\ 13565-905 São Carlos - SP, Brazil
}

Received: October 11, 2002; Revised: May 11, 2003

\begin{abstract}
The Gorsky Effect was investigated by internal friction measurements in the Nb-46 wt.\% $\mathrm{Ti}-\mathrm{H}$ system. The internal friction measurements were performed in a torsion pendulum with frequency of $3.8 \mathrm{~Hz}$ in a temperature range from 80 to $700 \mathrm{~K}$. An interpretation of the experimental data showed that the hydrogen diffusion coefficient D obeys the Arrhenius' law in the temperature interval of 300 to $700 \mathrm{~K}$, with $\mathrm{D}_{0}=7.4 \times 10^{-4} \mathrm{~cm}^{2} / \mathrm{s}$ and activation energy $\mathrm{E}=(0.102 \pm 0.005)$ $\mathrm{eV}$. At low temperatures, $\mathrm{D}_{\mathrm{o}}$ and $\mathrm{E}$ were found to be respectively $2.0 \times 10^{-4} \mathrm{~cm}^{2} / \mathrm{s}$ and $(0.063 \pm 0.005)$ $\mathrm{eV}$, with a deviation of the Arrhenius law of higher temperatures.
\end{abstract}

Keywords: alloys, anelasticity, diffusion, mechanical properties

\section{Introduction}

The diffusion coefficient for interstitial solutes in metallic systems is determined accurately by Elastic After Effect relaxation measurements, i.e., internal friction, ultrasonic attenuation and acoustic wave dispersion ${ }^{1,4}$.

The relaxation by diffusion is based in the lonely condition that the strain field produced by the applied stress (which can be of any symmetry) causes expansion or/and contraction in the crystalline lattice. The dilatation gradient acts as a driving force ${ }^{3}$ in each particle, promoting its diffusion. In the strain caused by bending a bar, the solute particles migrate from one side to other side in the sample. In the case of torsion vibration caused in polycrystals with inhomogeneous grain size, it causes radial strain components, making a cylindrical sample to contract and expand radially, enough for diffusion of defects from the center to the surface of the cylindrical sample and vice versa. The diffusion relaxation time $\tau$ is directly proportional to the square of the diameter of the sample and inversely proportional to the diffusion coefficient $\mathrm{D}$. The diffusion relaxation time is much higher than the ordering relaxation time and is given approximately by the relation $d^{2} / a^{2}$ (where $a$ is the lattice parameter and $d$ is the sample diameter $)^{5}$.
The torsional modulus $\mathrm{G}$ isn't pure in polycrystalline materials with inhomogeneous grains size as in isotropic materials (single crystals). Thus, a compression component of this heterogeneous compression module might exist. The inhomogeneous tension causes compression that give origin to the Gorsky Effect, that is, the G modulus isn't only torsional and it must have a compression component.

The Gorsky Effect due to hydrogen and deuterium longrange diffusion in niobium and vanadium was obtained by elastic after effect and internal friction measurements ${ }^{4,6}$. In spite of hydrogen and deuterium diffusion in metals and alloys being studied by several researchers ${ }^{4-8}$, the experimental data are not sufficient to understand the diffusion mechanisms of these atoms in these materials.

The metallic superconductors more thoroughly used in the moment are those that still use niobium and titanium as base materials. All the modern magnets also use Nb-Ti alloys. The great majority of the Nb-Ti superconductors industrially produced uses the Nb-46 wt. $\%$ Ti alloy as base material, whose fusion process, of high cost, require precise quality control to prevent the development of composition gradients.

The hydrogen present in this alloy causes embritlement,

*e-mail: betog@ @fc.unesp.br 
reason why it is of fundamental importance to know the metal-hydrogen interaction mechanisms.

In the present work the Gorsky Effect was investigated by internal friction measurements in the Nb-46 wt.\% Ti-H system using a torsion pendulum. The pre-exponential factors and activation energies of hydrogen in this alloy was found and interpolated over a wide temperature range.

\section{Experimental Details}

The Nb-46 wt.\% Ti alloy was produced by Materials Engineering Department of Chemical Engineering Faculty (FAENQUIL), Lorena (Brazil). This alloy was obtained by electron beam zone melting at pressure of less than $10^{-4}$ Torr. The typical chemical composition of the sample is shown in Table $1^{9,10}$.

The sample was cold-worked starting from a bar 350 $\mathrm{mm}$ long and $8 \mathrm{~mm}$ in diameter, to $50 \mathrm{~mm}$ long and $3 \mathrm{~mm}$ in diameter. To use these samples in internal friction measurements by torsion pendulum technique, we had to reduce its diameter to less that $1.5 \mathrm{~mm}^{10}$. Our samples were reduced in diameter by chemical polishing using a nitric and fluoridric acid solution.

Measurements were taken on samples at three different conditions: cold-worked; annealed in an ultra-high-vacuum furnace and doped with 0.5 at $\%$ of hydrogen. In the second situation, the sample was annealed in a VARIAN model FC 123 UHV furnace at $1073 \mathrm{~K}$ and $4.0 \times 10^{-8}$ Torr vacuum during $4 \mathrm{~h}$. In the third situation, the sample was doped with hydrogen by gas-charge method ${ }^{11}$. The hydrogen equilibrium solubility data for $\mathrm{Nb}-46 \mathrm{wt} . \% \mathrm{Ti}$ system used were obtained by Florêncio et al. ${ }^{12-13}$. The sample was charged at $1073 \mathrm{~K}$ by ninety minutes with 80 Torr of hydrogen partial pressure resulting about $0.5 \mathrm{wt} . \%$ of concentration.

The internal friction data were obtained using a torsion pendulum of the inverted Kê type ${ }^{14}$, with a heating rate of $1.0 \mathrm{~K} / \mathrm{min}$, in a pressure near $10^{-3}$ Torr and an oscillation frequency of about $3.8 \mathrm{~Hz}$. The oscillation decay data were collected automatically by 2 phototransistors connected to a computer. A laser beam was deflected by a mirror on the pendulum bar and the phototransistors were placed sideby-side in the path of the deflected beam near the center of oscillation so that the beam transit time was inversely proportional to the oscillation amplitude. The internal friction was obtained from the amplitude decay, which was determined by the ratio of the two measured transit time ${ }^{15}$.

\section{Experimental Results}

Figure 1 shows the internal friction as a function of temperature measured at frequency of $3.8 \mathrm{~Hz}$ for the $\mathrm{Nb}-46$ wt.\% Ti alloy in two conditions, cold-worked and annealed ${ }^{16}$. We can observe the effect caused by annealing.

Figure 2 shows the internal friction as a function of temperature for second and third conditions, measured with the same frequency. The lower curve is the annealed sample where we observe a complex relaxation structure composed by several Debye type peaks due to stress induced ordering of oxygen and nitrogen atoms present in the sample around $\mathrm{Nb}$ and $\mathrm{Ti}$ atoms of the metallic matrix ${ }^{16,17}$. In the upper curve, we observe an increase of the relaxation strength caused by introduction of hydrogen.

Using the same procedure as that of Cantelli et al. ${ }^{4}$ for flexural vibrations, we show the results obtained in torsion pendulum in the Fig. 3.

\section{Discussion}

The relaxation theory of diffusion formulated by Gorsky ${ }^{3}$ and developed by Alefeld et al. ${ }^{1,2}$ for cylindrical samples, is given by:

$$
\mathrm{Q}^{-1}(\mathrm{~T})=\frac{\Delta_{\mathrm{G}}}{\mathrm{T}} \sum_{\mathrm{n}=1,3,3, \ldots, \ldots}\left\{\frac{1}{n^{4}}\left(\frac{2 \omega \tau_{\mathrm{n}}}{1+\omega^{2} \tau_{n}^{2}}\right)\right\}
$$

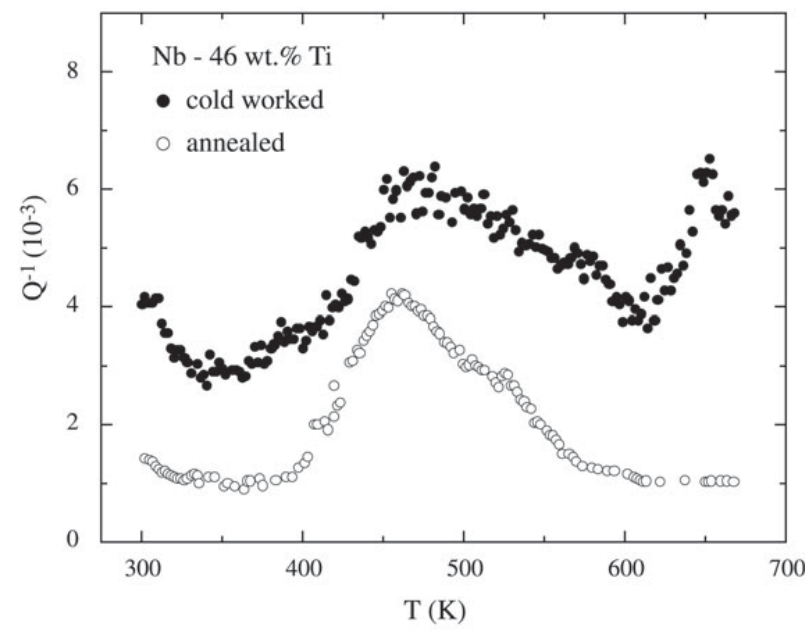

Figure 1. Internal friction as a function of temperature for the $\mathrm{Nb}-46$ wt.\% Ti sample, cold-worked and annealed, measured with frequency of $3.8 \mathrm{~Hz}$.

Table 1. Chemical Analysis of the Nb-46 wt.\% Ti alloy,10.

\begin{tabular}{lccccccccc}
\hline Elements & $\mathrm{Ta}$ & $\mathrm{Fe}$ & $\mathrm{Si}$ & $\mathrm{Ni}$ & $\mathrm{Cu}$ & $\mathrm{Al}$ & $\mathrm{O}$ & $\mathrm{N}$ & $\mathrm{C}$ \\
\hline Concentration (wt. ppm) & $<500$ & 67 & 65 & 93 & 6 & 29 & 500 & 126 & 44 \\
\hline
\end{tabular}


where: $\tau_{\mathrm{n}}=\frac{\mathrm{d}^{2}}{\mathrm{n}^{2} \pi^{2} \mathrm{D}^{2}}$

$\mathrm{d}$ is the sample diameter.

$\mathrm{D}$ is the diffusion coefficient of the migrant solute atoms.

Neglecting the contributions of the higher order terms in comparison to the first order term, we have:

$$
\mathrm{Q}^{-1}(\mathrm{~T})=\frac{\Delta_{\mathrm{G}}}{\mathrm{T}}\left(\frac{2 \omega \tau_{1}}{1+\omega^{2} \tau_{1}^{2}}\right)
$$

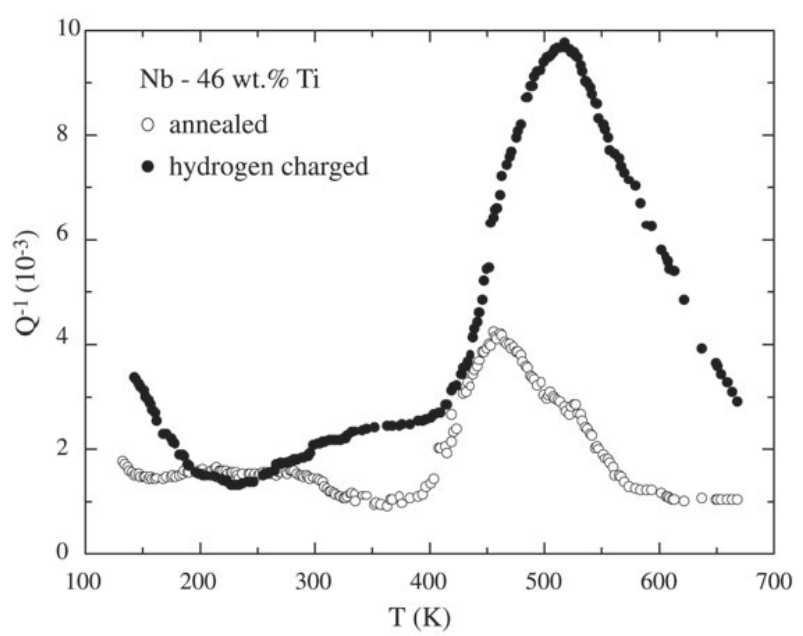

Figure 2. Internal friction as a function of temperature for the Nb-46 wt.\% Ti sample, annealed and hydrogen charged, measured with frequency of $3.8 \mathrm{~Hz}$.

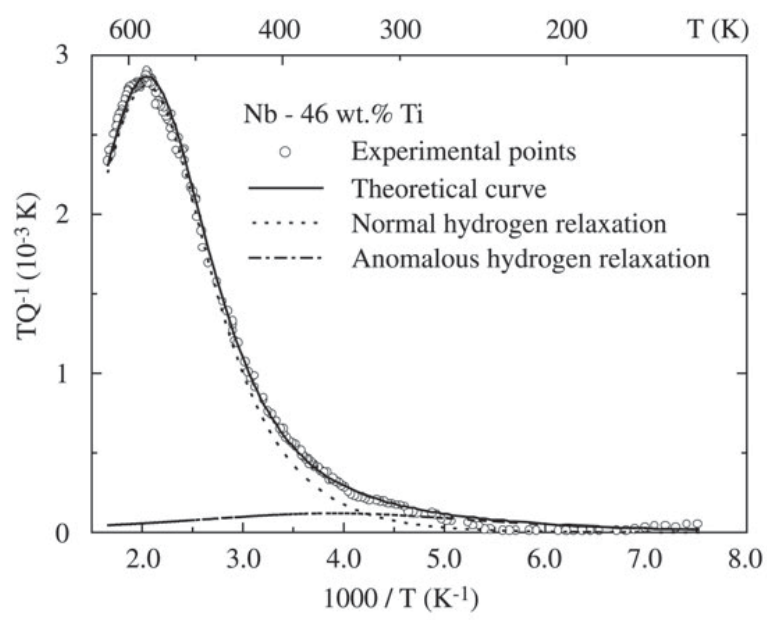

Figure 3. Internal friction $v s$. the inverse of the temperature for the Nb-46 wt.\% Ti sample, due to Gorsky Effect. where: $\tau_{1}$ is the diffusion relaxation time obtained by Arrhenius' equation, $\tau_{1}=\tau_{01} \exp (\mathrm{E} / \mathrm{kT})$, with $\mathrm{E}$ as the activation energy of migrant solute atoms and $\mathrm{k}$ as the Boltzmann constant.

The relaxation strength $\Delta_{\mathrm{G}}$ has different values for the hydrogen diffusion occurring above or below $300 \mathrm{~K} . \Delta_{\mathrm{G}>}$ is denoted as the relaxation strength at temperatures higher than $300 \mathrm{~K}$ and $\Delta_{\mathrm{G}<}$ as the relaxation strength at temperatures under $300 \mathrm{~K}$. Thus:

$$
\begin{aligned}
& \Delta_{\mathrm{G}>}=\frac{\mathrm{C}_{>} \mathrm{v}_{\mathrm{o}}}{\mathrm{T}_{\mathrm{p}>}} \frac{(\Delta \lambda)_{>}^{2}}{\mathrm{~J}_{\mathrm{u}}} \\
& \Delta_{\mathrm{G}<}=\frac{\mathrm{C}_{<} \mathrm{v}_{\mathrm{o}}}{\mathrm{T}_{\mathrm{p}<}} \frac{(\Delta \lambda)_{<}^{2}}{\mathrm{~J}_{\mathrm{u}}}
\end{aligned}
$$

where: $\mathrm{C}_{>}$and $\mathrm{C}_{<}$are the defect concentration at temperatures above or below $300 \mathrm{~K}$, respectively; $\mathrm{T}_{\mathrm{p}>}$ and $\mathrm{T}_{\mathrm{p}<}$ are the peak temperature for the peak located at the left or at the right side of $300 \mathrm{~K}$, respectively; $\mathrm{V}_{0}$ is the unit cell volume; $\mathrm{J}_{\mathrm{u}}$ is the unrelaxed elastic constant appropriated and $(\Delta \lambda)_{>}$and $(\Delta \lambda)_{<}$are the form factor of the migrant defects at temperatures above or below $300 \mathrm{~K}$, respectively.

For constant values of concentration, $\Delta_{\mathrm{G}>}$ and $\Delta_{\mathrm{G}<}$ are constant. The maximum value is obtained at the peak temperature $\mathrm{T}_{p}$ when $\omega \tau_{1}=\omega \tau_{01} \exp \left(\mathrm{E} / \mathrm{kT}_{\mathrm{p}}\right)=1$. Figure 3 shows the $\mathrm{TQ}^{-1} v s . \mathrm{T}^{-1}$. The results obtained are shown as a relaxation spectrum composed by two single relaxation peaks. The curve 1, located around $500 \mathrm{~K}$ shows the diffusion relaxation of hydrogen through equivalent sites and the curve 2 , located around $250 \mathrm{~K}$ shows the diffusion relaxation of hydrogen at low temperatures, with relaxation strength $5 \%$ of the curve 1 . This small peak is probably due to the hydrogen diffusion in other phase of the Nb-46 wt.\% Ti-H alloy, at low temperatures. Using the relations (2) and (3), the diffusion relaxation time as well as the hydrogen diffusion coefficient in the alloy were plotted as a function of inverse of temperature, shown in Figs. 4 and 5 respectively.

The diffusion coefficient is especially strongly reduced bellow $316 \mathrm{~K}$, where a decrease of the activation enthalpy is observed. This low-temperature anomaly, which is yet theoretically unexplained, is an indication for a change of the predominant microscopic diffusion mechanism. We observe that the continuous lines obtained by a linear regression of experimental data determine two diffusion coefficients. These continuous lines show an inflection point around $300 \mathrm{~K}$ showing that there are two different diffusion relaxation processes that occur below and above $316 \mathrm{~K}$.

Using the successive subtraction method ${ }^{16}$, the complex relaxation structure was decomposed in two peaks and consequently the activation energy of the process was deter- 


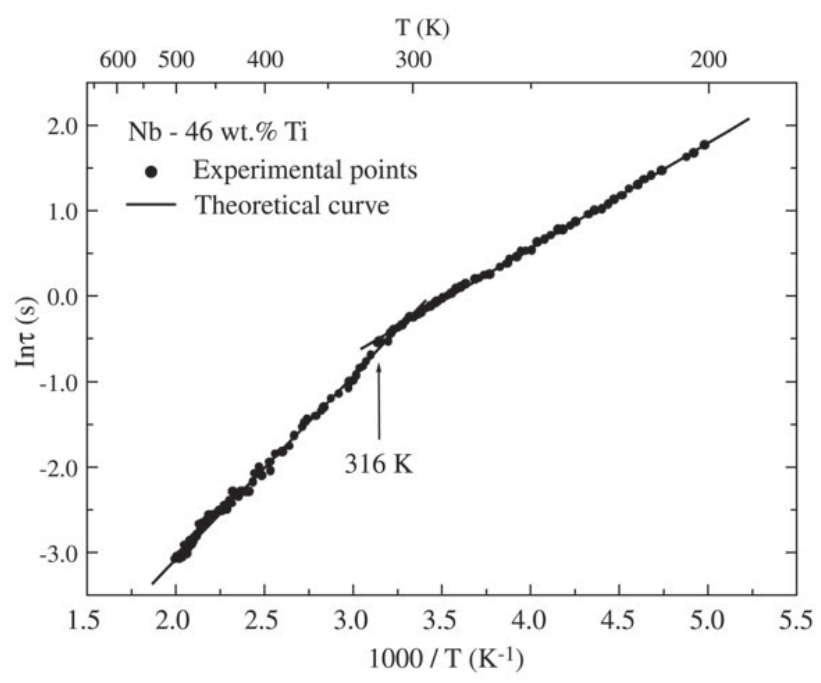

Figure 4. Diffusion relaxation time vs. inverse of the temperature for the Nb-46 wt.\% Ti sample, due to Gorsky Effect.

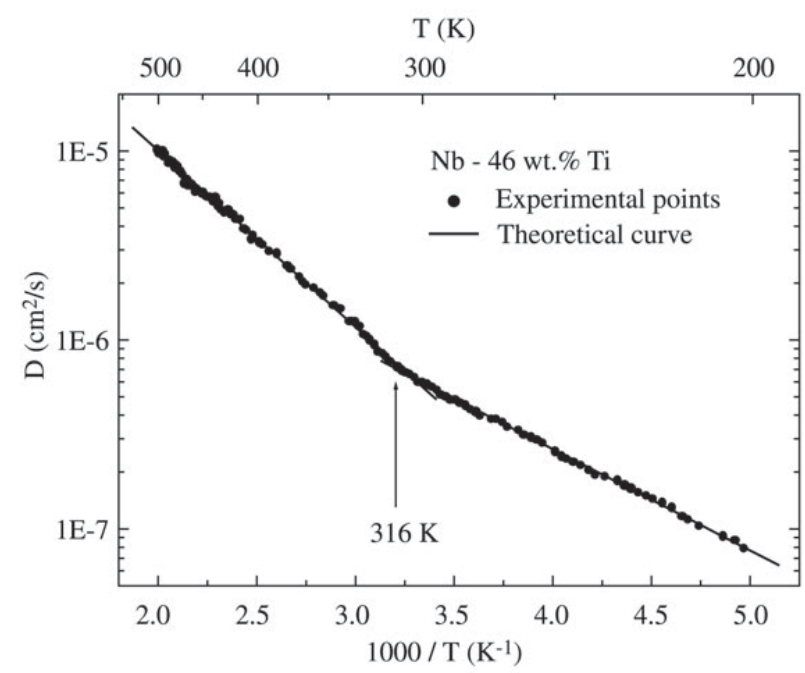

Figure 5. Diffusion coefficient $v s$. inverse of the temperature for the Nb-46 wt.\% Ti sample, due to Gorsky Effect.

Table 2. Experimental results obtained by several authors.

\begin{tabular}{lcccc}
\hline System & $\begin{array}{c}\mathrm{D}_{0} \\
\left(10^{-4} \mathrm{~cm}^{2} / \mathrm{s}\right)\end{array}$ & $\mathrm{E}(\mathrm{eV})$ & $\mathrm{T}(\mathrm{K})$ & Ref. \\
& 7.4 & 0.102 & $>300$ & this work \\
Nb-46 wt.\% Ti-H & 2.0 & 0.063 & $<300$ & this work \\
Nb-46 wt.\% Ti-H & 2.4 & 0.109 & $>235$ & 4 \\
Nb-H & 5.0 & 0.106 & $>300$ & 5 \\
Nb-H & 0.9 & 0.068 & $<300$ & 5 \\
Nb-H & 5.0 & 0.106 & $>273$ & 7 \\
Nb-H & 0.9 & 0.068 & $<273$ & 7 \\
Nb-H & & & & \\
\hline
\end{tabular}

mined. The main results are presented below:

$$
\begin{gathered}
E_{>}=(0.102 \pm 0.005) \mathrm{eV} \\
E_{<}=(0.063 \pm 0.005) \mathrm{eV} \\
t_{01}=(0.7 \pm 0.2) \times 10^{-3} \mathrm{~s} \\
t_{01}=(13.6 \pm 0.2) \times 10^{-3} \mathrm{~s} \\
\mathrm{D}^{>}=(7.4 \pm 0.2) \times 10^{-4} \mathrm{~cm}^{2} / \mathrm{s} \\
\mathrm{D}^{<}=(2.0 \pm 0.2) \times 10^{-4} \mathrm{~cm}^{2} / \mathrm{s}
\end{gathered}
$$

Our results are very close to those found in the literature ${ }^{4,-8}$, as showed in Table 2. The uncertainty is in the results found at low temperatures. As shown that the relaxation strength of the peak at low temperatures, $\Delta_{\mathrm{G}<}$ is about $5 \%$ of $\Delta_{\mathrm{G}>}$. For this reason, it is not attributed to the same diffusion relaxation process as that of $\Delta_{\mathrm{G}>}$. This anomalous peak at $250 \mathrm{~K}$ is proposed to explain the break in the diffusion coefficient. It can be attributed to a different diffusion process that occurs when a phase transformation at low temperatures, $\alpha \rightarrow \beta$, occur in such alloy doped with hydrogen ${ }^{18}$, probably an association with precipitation effects.

We conclude that the long-range diffusion of interstitial solutes in metallic alloys could be studied by internal friction measurements.

\section{Acknowledgements}

The authors thank to Departamento de Engenharia de Materiais of Faculdade de Engenharia Química de Lorena (DEMAR-FAENQUIL) by the samples; Conselho Nacional de Desenvolvimento Científico e Tecnológico (CNPq), Coordenação de Aperfeiçamento de Pessoal de Nivel Superior (Capes) and Fundação para o Desenvolvimento da UNESP (FUNDUNESP) for financial support; and Profs. M.M. Shukla and H. Tejima for the useful discussions.

\section{References}

1. Schaumann, G.; Völkl, J.; Alefeld, G. Physical Review Letters, v. 21, p. 891-893, 1968.

2. Alefeld, G.; Völkl, J.; Schaumann, G. Physica Status Solidi, v. 37, p. 337-345, 1970.

3. Gorsky, W.S. Physik Z. Sowjetunion, v. 8, p. 457-471, 1935.

4. Cantelli, R.; Mazzolai, F.M.; Nuovo, M. Physica Status Solidi, v. 34, p. 597-600, 1969.

5. Schaumann, G.; Völk1, J.; Alefeld, G. Physica Status Solidi, v. 42, p. 401-413, 1970.

6. Cantelli, R.; Mazzolai, F.M.; Nuovo, M. Journal of Physics and Chemistry of Solids, v. 31, p. 1811-1817, 1970.

7. Völkl, J.; Alefeld, G. Il Nuovo Cimento, v. 33B, p. 191-204, 1976. 
8. Völkl, J.; Alefeld, G. Hydrogen Diffusion in Metals, Chapter 5, p. 231-302. in: Nowick, A.S; Burton, J.J. Diffusion in Solids: Recent Developments, Academic Press, 1975.

9. Conti, R.A. Master Thesis, UNICAMP, Campinas (Brazil), 1984.

10. Florêncio, O. Ph.D. Thesis, USP-IFQSC, São Carlos (Brazil), 1986.

11. Fromm, E.; Hörz, G. International Metals Review, v. $5 / 6$, p. $269-311,1980$.

12. Florêncio, O.; Silva, J.R.G. Revista Latinoamericana de Metalurgia y Materiales, v. 5, p. 35-40, 1985.

13. Florêncio, O.; Cabral, F.A.O.; Ishikawa, T.T.; Silva,
J.R.G. Revista Latinoamericana de Metalurgia y Materiales, v. 5, p. 105-108, 1985.

14. Kê, T.S., Physical Review, v. 71, p. 533-541, 1947.

15. Grandini, C.R., Ph.D. Thesis, USP-IFQSC, São Carlos (Brazil), 1993.

16. Grandini, C.R.; Florêncio, O.; Botta Filho, W.J. submitted to Acta Materialia, 2003.

17. Florêncio, O.; Botta Filho, W.J.; Grandini, C.R.; Tejima, H. Jordão, J.A.R. Journal of Alloys and Compounds, v. 211/212, p. 37-41, 1994.

18. Hanada, S.; Ozeki, M.; Izumi, O. Metallurgical Transactions A, v. 16A, p. 789-795, 1985. 